Historic, Archive Document

Do not assume content reflects current scientific knowledge, policies, or practices. 



\section{CHOICE BULBS FOR FALL PLANTING}

BELLE ALLIANCE

CHRYSOLORA

COTTAGE MAID

FLAMINGO

KAISERKROON

MON TRESOR

PINK BEAUTY

PROSERPINE

PROSPERITY

QUEEN OF NETHERLANDS

ROSE LUISANTE

SIR THOMAS LIPTON

VERIIILLION BRILLIANT

WHITE HAWK

IMPERATOR RUBRUM

MR. VAN DER HOEFF

MURILLO

SALVATOR ROSA

TEA ROSE

BARON TONNAYE

BARTIGON

CLARA BUTT

CENTENAIRE

DREAM

FARNCOMBE SANDERS

KING HAROLD

LA TULIPE NOIR

PRIDE OF HAARLEM

PRINCE OF NETIERLANDS

PRINCESS ELIZABETH

REVEREND EWBANK

SALMON KING

INGLESCOMBE YELLOW

JOHN RUSKIN

SARAH BERNHARDT

THE FAWN

ABD-EL-KADER

APRICOT

FEU ARDENT

LE GRAND CONCURRENT

MARIE LOUISE

PANORAMA

TURENNE

VELVET KING

YELLOW PERFECTION
EARLY TULIPS

scarlet

golden yellow

bright pink

white and rosy red

scarlet edged yellow

fine ceep yellow

bright pink, white markings

satiny carmine rose

delicate pink

delicate satiny pink

deep pink

brilliant scarlet

vermillion scarlet

pure white

Doz.

$\$ 1.00$

1.00

1.00

1.00

1.25

1.50

2.00

1.25

1.00

1.00

1.00

1.25

1.25

1.00

DOUBLE TULIPS

$12^{\prime \prime} \quad$ glowing red

$13^{\prime \prime} \quad$ golden yellow

$11^{\prime \prime} \quad$ light pink

$10^{\prime \prime}$ beautiful deep rose

$12^{\prime \prime}$

yellow shaded apricot

DARWIN TULIPS

$25^{\prime \prime} \quad$ vivid pink, light margin

$24^{\prime \prime} \quad$ large fiery red

$20^{\prime \prime} \quad$ soft salmon pink

$32^{\prime \prime} \quad$ deep rose flushed claret

$25^{\prime \prime} \quad$ beautiful soft lilac

$27^{\prime \prime} \quad$ brilliant scarlet, white base

$20^{\prime \prime} \quad$ glowing ruby crimson

$25^{\prime \prime} \quad$ maroon black

$26^{\prime \prime} \quad$ old rose

$30^{\prime \prime} \quad$ scarlet and salmon rose

$22^{\prime \prime} \quad$ soft delicate rose

$20^{\prime \prime} \quad$ lavender, shaded silver

$22^{\prime \prime}$

salmon, white center

1.00

1.25

.75

1.00

1.00

.75

.75

.75

.85

.85

.75

.85

1.25

.75

.85

.85

.75

.85

\section{LATE BLOOMING TULIPS}

$18^{\prime \prime} \quad$ canary yellow

$18^{\prime \prime} \quad$ apricot pink edged lemon

$12^{\prime \prime}$

rose buff

rosy fawn

.75

.75

.75

.75

BREEDER TULIPS

$30^{\prime \prime} \quad$ dark bronze

$26^{\prime \prime} \quad$ apricot flushed orange

$28^{\prime \prime}$

fiery red, mahogany shading

1.00

1.00

1.50

1.00

1.00

salmon with yellow

dark orange red

violet, yellow margin

rich purple_violet

yellowish olive

1.00

1.50

1.00
Hun.

$\$ 6.00$

6.00

6.00

6.00

9.00

10.00

14.00

9.00

6.00

6.00

7.00

8.00

9.00

6.00

6.00

8.00

5.00

6.00

6.00

5.00

5.00

5.00

6.00

6.00

5.00

6.00

8.00

5.00

6.00

6.00

5.00

6.00

5.00

5.00

5.00

5.00

7.50

7.50

10.00

7. 0

7.50

7.50

7.50

10.00

7.50 


\section{NARCISSUS}

In limited quantities, of excellent quality, double nose

Doz. Hun.

DOUBLE VON SION

rich golden yellow

deep golden yellow

GOLDEN SPUR

KING ALFRED

golden, deeply frilled trumpet
white perianth, orange-red cup

LUCIFER

POETAZ LAURENS KOSTER

pure white, orange-yellow cup

POET'S NARCISSUS

white, orange crown edged scarlet

SIR WATKIN

sulphur, deep yellow cup

VICTORIA BICOLOR

creamy white, yellow trumpet

ASSORTED VARIETIES FOR NATURALIZING (single and double)

$\$ 2.00 \quad \$ 15.00$

$2.00-15.00$

$2.00 \quad 15.00$

$3.75 \quad 28.00$

$2.00 \quad 15.00$

$2.00 \quad 14.00$

$1.25 \quad 9.00$

$2.00 \quad 15.00$

$2.00 \quad 15.00$

$1.00 \quad 10.00$

$3.75 \quad 28.00$

HYACINTHS-1st Size Bulbs

CITY OF HAARLEM

deep yellow

brilliant carmine, early

dark pink, large spike

sky blue, shaded violet

LADY DERBY

LA GRANDESSE

LA VICTOIRE

GERTRUDE

GRAND MAITRE

JOHAN

light blue, very fine

bright rose pink

large bells of snow white brilliant scarlet

deep rose fiushed white

2.75

20.00

deep crimson

light blue, very fine

bright rose pink

LA GRANDESSE
MARCONI
QUEEN OF PINKS

large bells of snow white deep rose fiushed white brilliant pink

1.75

12.50

BLANCHEUR A MERVILLE GENERAL DE WET

JOHAN

HYACINTHS - 3rd Size Bulbs

pure white

beautiful bright pink

light blue, very fine

\section{CROCUS}

.60

4. 00

light blue, very fine

striped, large flower

yellow

pure white

deep purple, large

pure white

heavenly blue

bright blue

GRAPE HYACINTH

SCILLA SIBERICA

LILY OF THE VALLEY

LILIUM CANDIDUM

“ REGALE

Lilium speciosum album

“ auratum

، “ platyphyllum

“ speciosum magnificum

$30 \phi$ each

$75 \%$ ،
3.00

3.50

5.00

$\begin{array}{rr}.75 & 5.00 \\ 3.00 & 25.00\end{array}$

$7.50 \quad 60.00$

JAPANESE LILIES - Spring Delivery

$\begin{array}{ll}\text { pure white } & 60 \% \text { each } \\ \text { white, gold-banded } & 45 \% \text { “ } \\ \text { “ yellow-spotted } & 45 \% \text { “ } \\ \text { carmine, white margin } & 45 \% \text { “ } \\ \text { VIO } & \\ \text { VIORO }\end{array}$

$6.00 \quad 50.00$

$4.50 \quad 35.00$

$4.50 \quad 35.00$

$4.50 \quad 35.00$

A highly concentrated plant food scientifically prepared. For all purposes where a fertilizer is used, it has given satisfactory results.

For garden planis it should be thoroughly mixed in the soil before planting. For house plants it should be mixed in the soil before potting, and added on the surface just before the blooming period. It produces wonderful velvety lawns, and has stood the test during the past summer. Full directions in the "How to Use" booklet in the top of each bag.
$100 \mathrm{lb}$. bag
$\$ 6.00$
50 “" “
3.75
$25 \mathrm{lb}$. bag
$\$ 2.00$
5 “" "
.60 